\title{
Design of a Secure Dynamic Identity Authentication Scheme for Health Internet of Things
}

\author{
Chengqi Wang ${ }^{1}$, Xiao Zhang ${ }^{1, *}$, Lijia Xie ${ }^{1}$ and Zhiming Zheng ${ }^{1}$ \\ ${ }^{1}$ Key Laboratory of Mathematics, Informatics and Behavioral Semantics, Ministry \\ of Education, and School of Mathematics and Systems Science, Beihang \\ University, Beijing 100191, China \\ *09621@buaa.edu.cn
}

\begin{abstract}
To satisfy the security requirements of patients' privacy and data's security for health Internet of Things (IoT), various authentication schemes are proposed as guaranteed countermeasures. In particular, Wang et al. built an identity-based authentication scheme with extended Chebyshev chaotic maps. Nevertheless, considering service misuse attack and Denial-of-Service attack, Wang et al.'s method works inadequately. Also it is insufficient to provide efficient password change phase, fast error detection and session key agreement. As a remedy, we propose a novel dynamic identity authenticated key agreement scheme. Our scheme achieves resistance to the known attacks in order to meet the desirable security requirements. Furthermore, the presented scheme practically enables both user revocation/re-registration and biometric information protection, which are significant features ignored by most previous schemes. We confirm the effectiveness of our scheme via comprehensive comparisons in terms of resistance, functionality and performance.
\end{abstract}

Keywords: Health Internet of Things, Authentication, Key agreement, Biometrics

\section{Introduction}

As a pervasive infrastructure, Internet of Things (IoT) has been enhanced in the services' quality due to the advances in the information technology [1-2]. Recently, health IoT becomes more popular as emerging healthcare applications which are equipped with communication capabilities and contributes to more electronic medical services for example clinical diagnosis and health records. Thus a convenient way for communication between doctors and patients over public channels is able to be established [3-4]. Note that health IoT is almost implemented and patients' information is transmitted over the public networks. In other words, hackers, spywares and other threats in the public networks migrate to the health IoT. When facing multiple kinds of attacks, how to ensure the security and privacy of transmitted information becomes a great challenge [5-6]. In response, many authentication algorithms have been proposed to guarantee the secure communication between remote participants [7-12]. According to applied evidences, existing proposals can be separated into two categories, namely, certificate-based and identity-based [13-16]. Nevertheless, without high requirements of both computational resource and storage space, identity-based authentication schemes are now more generally adopted than certificate-based ones in the health IoT in order to provide convenient health-care services.

Besides, there are still some vulnerabilities for two-factor identity-based authentication algorithms which apply tokens and passwords to guarantee the security [17-19]. Specifically, patients usually feel difficult to use random and long passwords. But short

Received (January 17, 2017), Review Result (August 22, 2017), Accepted (August 30, 2017) 
passwords can be easily cracked by dictionary attack because of low entropy. Moreover, common side channel attacks, for instance SPA and DPA, makes it feasible to acquire sensitive information saved in smart cards [20]. To overcome these weaknesses, many research papers focus on combination of passwords, tokens and biometrics, namely threefactor, to improve the security [21-22]. Since biometric information is unique and unforgeable [23-24], users are not required to remember their biometric information. However, without professional recognition mechanisms, imprinted biometric characteristics which may not be accepted for the same user increase the probability of rejection [25-26]. Thus, more work about authenticated key agreement algorithms in health IoT needs to be built with three-factor.

Furthermore, Wang et al. [27] recently proposed an identity-based authentication scheme for health IoT to meet the problems of previous proposals. Unfortunately, based on cryptanalysis provided in this paper, we identify that their algorithm is still vulnerable to service misuse attack, Denial-of-Service attack and flaws exist in the password change phase. Also we show that Wang et al.'s scheme fails to achieve fast error detection and session key agreement. In addition, there is no consideration of the revocation or reregistration phase in their scheme. To address these issues, we propose a three-factor identity-based authenticated key agreement scheme, which is built upon extended chaotic maps. Our scheme improves Wang et al.'s proposal and satisfies desirable requirements. Compared with other related algorithms, with the same level of overhead, our scheme guarantees more properties and functionalities, especially biometric information protection.

Remaining of this paper is organized as below. First, we briefly introduce fuzzy extractor, extended Chebyshev chaotic map and threat assumptions applied in our proposal during next section. Section 3 and Section 4 reviews the Wang et al.'s scheme and discusses the weaknesses of their proposal, respectively. Next, we specify the details of our proposal in Section 5. Then section 6 provides the resistance, functionality and performance analysis of our algorithm. Lastly, we present our conclusion in Section 7.

\section{Preliminaries}

During this section, we basically describe the fuzzy extractor, extended Chebyshev chaotic map and threat assumptions for better understanding of our scheme.

\subsection{Fuzzy Extractor}

As can be seen in Fig. 1, mechanism of fuzzy extractor includes two procedures.

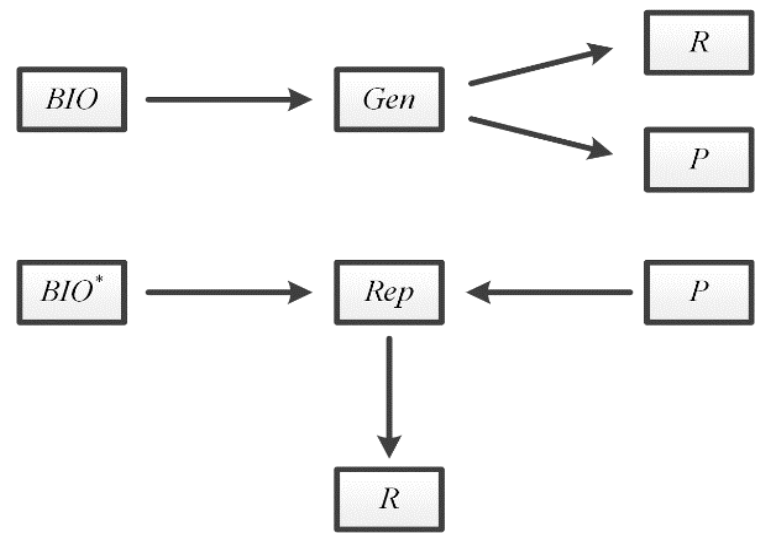

Figure 1. Mechanism of Fuzzy Extractor

Specifically, procedure Gen means a probabilistic generation function used to extract user's original biometrics input BIO. Its outputs cover nearly random binary string 
$R \in\{0,1\}^{l}$ and auxiliary binary string $P \in\{0,1\}^{*}$. Analogously, procedure Rep denotes a deterministic reproduction function used to recover $R$ with two inputs, namely, biometrics input $B I O^{*}$ and corresponding string $P$. When $\operatorname{Gen}(B I O) \rightarrow\langle R, P\rangle$ and $\operatorname{dis}\left(B I O, B I O^{*}\right) \leq t$, we obtain $\operatorname{Rep}\left(B I O^{*}, P\right)=R$, in which error-tolerant technology makes it dependable to retrieve the string $R$ from user's biometrics input $B I O^{*}$. Due to space constraints, we omit the details about the fuzzy extractor specified in the literature [25-26].

\subsection{Extended Chebyshev Chaotic Map}

In details, Chebyshev chaotic map $T_{n}(x)$ can be defined by following formula.

$$
T_{n}(x)=\cos n \theta \text {, }
$$

where $x=\cos \theta$ [28].

Furthermore, $T_{n}(x)$ 's recurrence formula is defined as follows.

$$
T_{n}(x)=2 x T_{n-1}(x)-T_{n-2}(x),
$$

in which for any natural number $n \geq 2, T_{0}(x)=1$ and $T_{1}(x)=x$.

As we know, Chebyshev chaotic map satisfies semi-group and commutative property under composition, namely, $T_{r}\left(T_{s}(x)\right)=T_{s}\left(T_{r}(x)\right)$. The proof can be specified as follows.

$$
T_{r}\left(T_{s}(x)\right)=\cos \left(r \cdot \cos ^{-1}\left(\cos \left(s \cdot \cos ^{-1}(x)\right)\right)\right)=\cos \left(r s \cdot \cos ^{-1}(x)\right)=T_{s r}(x)=T_{s}\left(T_{r}(x)\right),
$$

in which for any natural number $s$ and $r$.

Zhang [29] enhanced Chebyshev chaotic map in 2008. This paper [29] proved that the semi-group and commutative property under composition still hold when the interval extends to $(-\infty,+\infty)$. Hereby, we present the extended Chebyshev polynomial as follows.

$$
T_{n}(x) \equiv\left(2 x T_{n-1}(x)-T_{n-2}(x)\right) \bmod p,
$$

in which $n \geq 2, x \in(-\infty,+\infty)$ and $p$ is any large prime number. Similarly, $T_{r}\left(T_{s}(x)\right)=T_{r s}(x)=T_{s}\left(T_{r}(x)\right) \bmod p$ also holds.

There are two hard problems for extended Chebyshev polynomials [30], which we specify here as follows.

ECDLP: given $x, y$ and $p$, finding an integer $r$ which satisfies $y=T_{r}(x) \bmod p$ is hard.

ECDDHP: given $T_{r}(x), T_{s}(x), T_{z}(x)$ and $x$, determining whether $T_{r s}(x)=T_{z}(x) \bmod p$ holds is hard.

\subsection{Threat Assumptions}

We take side-channel attacks [20] and Dolev-Yao threat model [31] into consideration. And we list threat assumptions as below.

1. Attacker $E$ might be a malicious user or an outsider in the health IoTs.

2. Attacker $E$ eavesdrops on all transmitted messages between user $U_{i}$ and server $S$ via a public channel.

3. Attacker $E$ enables to delete, modify, reroute and resend all eavesdropped messages.

4. Attacker $E$ is able to extract sensitive information from stolen or obtained smart card $S C_{i}$ by measuring the power consumption. 


\section{Review of Wang et al.'s Scheme}

Wang et al.'s scheme [27] proceeds in four phases, namely, registration phase, login phase, verification phase and password change phase, respectively. Without loss of generality, server $S$ selects a hash function $h(\cdot)$, generates a random variable $x \in[-1,1]$, selects a private key $s$ and publishes $\left\{x, T_{s}(x), h(\cdot)\right\}$.

\subsection{Registration Phase}

1. The new user $U_{i}$ chooses his identity $I D_{i}$ and password $P W_{i}$. Then $U_{i}$ generates a random integer $t_{i}$, computes $W_{i}=P W_{i} \oplus t_{i}$, and sends his registration request $\left\{I D_{i}, W_{i}\right\}$ to the server $S$ via a secure channel.

2. Upon receiving this registration request, $S$ calculates $H_{i}=h\left(s \| I D_{i}\right)$ and $n_{i}=h\left(W_{i} \| I D_{i}\right) \oplus H_{i}$, respectively. And $S$ sends a smart card $S C_{i}$ to user $U_{i}$, which includes $\left\{n_{i}, x, T_{s}(x)\right\}$ via a secure channel.

3. After obtaining the $S C_{i}, U_{i}$ computes $N_{i}=h\left(I D_{i} \| P W_{i}\right) \oplus n_{i} \oplus h\left(W_{i} \| I D_{i}\right)$. Lastly, user $U_{i}$ stores $\left\{N_{i}, x, T_{s}(x)\right\}$ into his smart card $S C_{i}$.

\subsection{Login Phase}

1. $U_{i}$ inserts his $S C_{i}$ into a smart card reader. And then $U_{i}$ inputs his identity $I D_{i}$ and password $P W_{i}$.

2. $S C_{i}$ generates a random number $k$, and calculates $H_{i}=N_{i} \oplus h\left(I D_{i} \| P W_{i}\right)$, $Z=T_{k}\left(T_{s}(x)\right), \quad C I D_{i}=I D_{i} \oplus h\left(Z \| T_{1}\right), \quad C=T_{k}(x), \quad$ and $\quad V_{i}=h\left(C I D_{i}\|C\| H_{i}\|Z\| T_{1}\right)$, respectively.

3. $U_{i}$ sends his login request $M_{1}=\left\{C I D_{i}, C, V_{i}, T_{1}\right\}$ to the server $S$ via a public channel.

\subsection{Verification Phase}

1. When obtaining a login request from $S C_{i}, S$ validates whether $T_{2}-T_{1} \leq \Delta T$ holds.

2. If it inequality satisfies, $S$ computes $Z=T_{s}(C), I D_{i}=C I D_{i} \oplus h\left(Z \| T_{1}\right)$, $V_{i}{ }^{\prime}=h\left(C I D_{i}\|C\| H_{i}\|Z\| T_{1}\right)$, respectively. And $S$ verifies whether $V_{i}$ is consistent with $V_{i}$. Otherwise, $S$ refuses this login request.

3. If they match, $S$ calculates $\lambda=h\left(H_{i}\left\|C I D_{i}\right\| V_{i}\left\|T_{1}\right\| T_{2} \| Z\right)$ and $V_{S}=h\left(\lambda\left\|H_{i}\right\| T_{1}\left\|T_{2}\right\| Z\right)$, respectively. And $S$ issues his authentication request $M_{2}=\left\{V_{S}, T_{2}\right\}$ to the user $U_{i}$. Otherwise, $S$ stops this session.

4. After receiving this authentication request, $S C_{i}$ firstly checks whether $T_{2}-T_{1} \leq \Delta T$ holds. And $S C_{i}$ retrieves $\lambda=h\left(H_{i}\left\|C I D_{i}\right\| V_{i}\left\|T_{1}\right\| T_{2} \| Z\right)$ and $V_{S}{ }^{\prime}=h\left(\lambda\left\|H_{i}\right\| T_{1}\left\|T_{2}\right\| Z\right)$, respectively. Then $S C_{i}$ checks whether $V_{S}{ }^{\prime}=V_{S}$ holds. If it holds, $S$ is authenticated by $U_{i}$ successfully. Otherwise, $U_{i}$ refuses server $S$ 's authentication request.

\subsection{Password Change Phase}

1. $U_{i}$ inputs his identity $I D_{i}$, old password $P W_{i}$ and new password $P W_{i}^{*}$, respectively. 
2. $S C_{i}$ computes $N_{i}^{*}=N_{i} \oplus h\left(I D_{i} \| P W_{i}\right) \oplus h\left(I D_{i} \| P W_{i}^{*}\right)$.

3. $S C_{i}$ replaces $N_{i}$ with $N_{i}^{*}$ in the memory of his smart card $S C_{i}$.

\section{Cryptanalysis of Wang et al.'s Scheme}

Wang et al.'s scheme ensures the user anonymity and the forward confidentiality. Their scheme hereby performs efficient defense towards server spoofing attack and insider attack, yet it is insufficient to prevent the service misuse attack. In addition, input verification has not been not well considered in the design of their proposal, which may result in Denial-of-Service attack and weakness during password change phase. Furthermore, Wang et al.'s scheme fails to concern the fast error detection, session key agreement and user revocation/re-registration.

\subsection{Service Misuse Attack}

In Wang et al.'s scheme, server $S$ maintains the private key $s$ and random variable $x$ without keeping the password tables or verifiers for all registered users. Thereby, Wang et al.'s scheme is resilient against the stolen verifier attack. Nevertheless, when facing the service misuse attack, Wang et al.'s scheme works inadequately due to the exposure of several secret parameters among non-registered users. Suppose that a legal user $U_{i}$ wants to misuse the medical services of health IoT for $n$ non-registered users $U_{j}$, where $j=1,2, \cdots, n$. The user $U_{i}$ shares the secret parameters pair $\left\{I D_{i}, P W_{i}\right\}$ with $U_{j}$ so that $U_{j}$ has the knowledge of $\left\{I D_{i}, P W_{i}\right\}$. As a result, $U_{j}$ is able to login to the server $S$ anytime, remaining concealed towards the server. The details of service misuse attack are described as follows. Each non-registered user $U_{j}$ generates the corresponding random number $k j$, and computes $H_{i j}=N_{i j} \oplus h\left(I D_{i} \| P W_{i}\right) \quad, \quad Z_{j}=T_{k j}\left(T_{s}(x)\right) \quad, \quad C I D_{i j}=I D_{i} \oplus h\left(Z_{j} \| T_{1 j}\right)$, $C_{j}=T_{k j}(x)$ and $V_{i j}=h\left(C I D_{i j}\left\|C_{j}\right\| H_{i j}\left\|Z_{j}\right\| T_{1 j}\right)$, respectively, with the knowledge of secret parameters $I D_{i}$ and $P W_{i}$, where $T_{l j}$ is $U_{j}$ 's current timestamp and $j=1,2, \cdots, n$. After receiving $U_{j}$ 's login request, server $S$ checks the verification information $V_{i}$ with private key $s$. Lastly, server $S$ allows the all non-registered users who knows secret parameters $\left\{I D_{i}, P W_{i}\right\}$ misuse attack,

\subsection{Denial-of-Service Attack}

The Denial-of-Service (DoS) attack, a major menace of service unavailability, is able to interrupt or suspend the services of a host indefinitely. Wang et al.'s scheme does not enable input verification of old password during the password change phase. As a consequence, attacker $E$ is able to get a temporary access to $U_{i}$ 's smart card $S C_{i}$ by launching the DoS attack. Specifically, attacker $E$ first inserts the smart card $S C_{i}$ to initiate the password change procedure. Then he inputs two distinct random passwords $P W_{a}$ and $P W_{a}{ }^{*}$ as old password and new password, respectively. Smart card $S C_{i}$ calculates $N_{i}^{*}=N_{i} \oplus h\left(I D_{i} \| P W_{a}\right) \oplus h\left(I D_{i} \| P W_{a}^{*}\right)$ and then replaces $N_{i}$ with $N_{i}^{*}$. In this way, it is clear that attacker $E$ successfully updates $N_{i}$, enabling user $U_{i}$ to compute $V_{i}$ to generate a valid login request $\left\{C I D_{i}, C_{i}, V_{i}, T_{1}\right\}$. Yet, $U_{i}$ fails to achieve a correct verification information $V_{i}$ with his valid password $P W_{i}$ as $P W_{a} \neq P W_{i}$. Smart card $S C_{i}$ calculates $H_{i}^{*}=N_{i} \oplus h\left(I D_{i} \| P W_{a}\right) \oplus h\left(I D_{i} \| P W_{a}^{*}\right) \oplus h\left(I D_{i} \| P W_{i}\right)$ 


$$
\begin{aligned}
& H_{i}^{*}=h\left(s \| I D_{i}\right) \oplus h\left(I D_{i} \| P W_{a}\right) \oplus h\left(I D_{i} \| P W_{a}^{*}\right) \quad \text { Furthermore, } S C_{i} \text { computes } \\
& V_{i}^{*}=h\left(C I D_{i}\|C\| H_{i}^{*}\|Z\| T_{1}\right) \text { and submits his login request }\left\{C I D_{i}, C, V_{i}^{*}, T_{1}\right\} \text { to the server } S .
\end{aligned}
$$
While receiving $U_{i}^{\prime}$ 's login request, server $S$ calculates $Z=T_{s}(C), I D_{i}=C I D_{i} \oplus h\left(Z \| T_{1}\right)$ $H_{i}{ }^{\prime}=h\left(s \| I D_{i}\right)$, and $V_{i}{ }^{\prime}=h\left(C I D_{i}\|C\| H_{i}{ }^{\prime}\|Z\| T_{1}\right)$, respectively. Thus this login request is considered invalid by server $S$, since $P W_{a} \neq P W_{a}^{*}, H_{i}^{*} \neq H_{i}{ }^{\prime}$ and $V_{i}^{*} \neq V_{i}{ }^{\prime}$. Attacker $E$ changes the password $P W_{i}$ without knowing the user's credentials like identity or old password, making service unavailable to users. To avoid this problem, we add the input verification of old password in our proposal.

\subsection{Weakness During Password Change Phase}

Due to inefficient password change phase, user $U_{i}$ may make a single mistake and cause the Denial-of-Service by himself. Since user $U_{i}$ may forget the password or just type wrongly by accident, thus resulting an incorrect old password input $P W_{e}$. Namely, user $U_{i}$ inputs an incorrect old password $P W_{e}$ and a new password $P W_{i}^{*}$ to update $P W_{i}$. Then smart card $S C_{i}$ calculates $N_{i}^{*}=N_{i} \oplus h\left(I D_{i} \| P W_{e}\right) \oplus h\left(I D_{i} \| P W_{i}^{*}\right)$ and achieve the updating with $N_{i}^{*}$. Hence, $U_{i}$ is unable to login to the remote server $S$ with his new password $P W_{i}^{*}$ as $P W_{e} \neq P W_{i}$, leading to the denial of service. It becomes clear why password change phase of Wang et al.'s scheme works inefficiently.

\subsection{No Fast Error Detection}

It is necessary to enforce fast error detection, namely, smart card $S C_{i}$ is expected to timely examine the incorrect passwords. During the login phase of Wang et al.'s scheme, $S C_{i}$ fails to detect the errors immediately. In other words, user $U_{i}$ inserts the smart card $S C_{i}$ and inputs mistaken information, namely, inaccurate identity $I D_{e}$ or incorrect password $P W_{e}$. Without fast error detection, $S C_{i}$ executes the following procedures as planned but cannot detect the errors locally. Then smart card $S C_{i}$ submits $U_{i}$ 's login request to server $S$. However, it doesn't work due to wrong inputs. With the above problem unsolved, fast error detection is urged to be added during the login phase.

\subsection{No Session Key Agreement}

Note that Wang et al.'s scheme does not perform the process of session key agreement. Hereby, user $U_{i}$ and server $S$ are incapable of establishing a session key used for subsequent communication. In order to provide the session key agreement, we add the corresponding procedure to generate the session keys in our proposal.

\subsection{No User Revocation/Re-registration Phase}

Moreover, Wang et al.'s scheme lacks user revocation/re-registration. As a result, when smart card $S C_{i}$ of the user $U_{i}$ is stolen or lost, $U_{i}$ cannot revoke his privilege or re-register. To promote the functionality, we correspondingly design the revocation/re-registration phase, which meets the requirements in the Health IoT. The details are specified in the following section.

\section{The Proposed Scheme}

Benefit from cryptanalysis of Wang et al.'s scheme, we present a novel robust biometrics based authentication and key agreement proposal for Health IoT. The proposed scheme has do several improvements on the Wang et al.'s scheme, which we specify here as follows: 1) it enables the resilience to service misuse attack by adding login records in 
the memory of server, 2) it applies the biometric information to enhance the entropy of secret parameters, 3) it enforces the input verification to achieve the defense against the DoS attack, 4) it leverages the fast error detection and session key agreement to guarantee better performance, and 5) it designs an additional revocation/re-registration phase to meet the requirements of users. Then in the following subsections, we demonstrate our proposal in five aspects, namely, registration phase, login phase, authentication phase, password change phase and user revocation/re-registration phase.

Without loss of generality, two participants are presented here, namely, user $U_{i}$ and server $S$. All notations applied in our scheme are listed in Table 1. In the initialization of medicine system, server $S$ first selects a hash function $h(\cdot)$. Next, server $S$ generates a pre shared key $s$ and a random variable $x \in[-1,1]$ for the later calculation. Finally, $S$ calculates $T_{s}(x)$ and publishes $\left\{x, T_{s}(x), h(\cdot)\right\}$.

Table 1. Symbols and Notions in Our Scheme

\begin{tabular}{|c|c|}
\hline Symbol & Notion \\
\hline$U_{i}, S, E$ & $i$ th user, server and adversary \\
\hline$S C_{i}, I D_{i}$, & $U_{i}$ 's smart card and $U_{i}^{\prime}$ s identity \\
\hline$A I D_{i}$ & $U_{i}$ 's dynamic identity \\
\hline$P W_{i}, B I O_{i}$ & $U_{i}$ 's password and $U_{i}$ 's biometric information \\
\hline$R_{i}$, & $U_{i}$ 's nearly random binary string \\
\hline$P_{i}$ & $U_{i}$ 's auxiliary binary string \\
\hline$s, x$ & Pre shared key and random variable \\
\hline$h(\cdot), \oplus, \|$ & Hash function, XOR operation and concatenation operation \\
\hline
\end{tabular}

\subsection{Registration Phase}

User $U_{i}$ and server $S$ perform the registration phase via a secure channel, in which registration phase is shown in the Figure 2 and details are specified as follows.

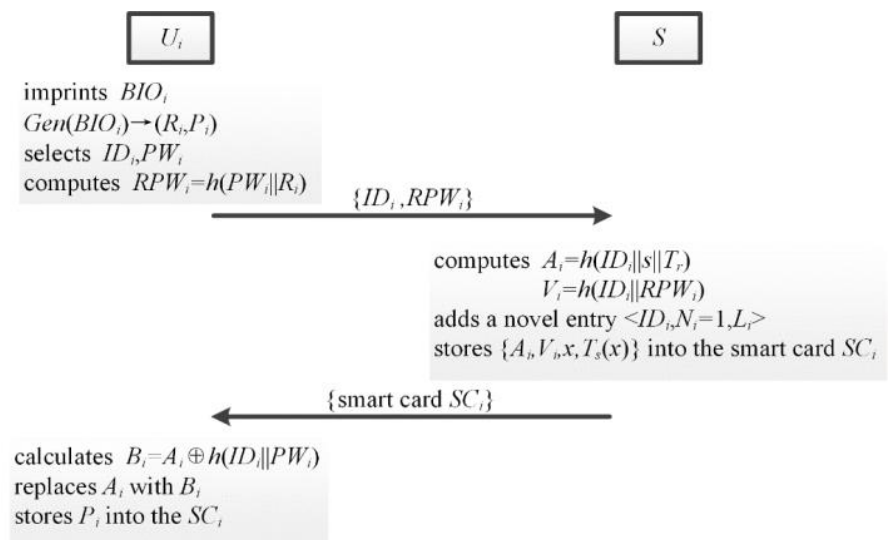

Figure 2. The Registration Phase

1. User $U_{i}$ first imprints his biometrics $B I O_{i}$ on the sensor. Next sensor helps user $U_{i}$ sketches his $B I O_{i}$, execute $\operatorname{Gen}\left(B I O_{i}\right) \rightarrow\left(R_{i}, P_{i}\right)$, and stores $P_{i}$, respectively. Then $U_{i}$ chooses his identity $I D_{i}$ and password $P W_{i}$, and computes $R P W_{i}=h\left(P W_{i} \| R_{i}\right)$. Finally, he submits his registration request $\left\{I D_{i}, R P W_{i}\right\}$ to the server $S$ via a secure channel. 
2. Upon receiving this registration request, $S$ calculates $A_{i}=h\left(I D_{i}\|s\| T_{r}\right)$ and $V_{i}=h\left(I D_{i} \| R P W_{i}\right)$, where $T_{r}$ is registration time. And $S$ adds a novel array $\left\langle I D_{i}, N_{i}=1, L_{i}\right\rangle$ to the server's database, in which $N_{i}$ means the number of user registration and $L_{i}$ records the login status of $U_{i}$, respectively. Particularly, if $U_{i}$ is logged-into the server $S$, $L_{i}$ is set to 1 . Otherwise, ${ }^{L_{i}}$ is set to 0 .

3. $S$ sends a smart card $S C_{i}$ to $U_{i}$, which includes $\left\{A_{i}, V_{i}, x, T_{s}(x)\right\}$ over a secure channel.

4. After receiving the $S C_{i}, U_{i}$ calculates $B_{i}=A_{i} \oplus h\left(I D_{i} \| P W_{i}\right)$. Then $U_{i}$ replaces $A_{i}$ with $B_{i}$ and further stores $P_{i}$ into the $S C_{i}$.

\subsection{Login Phase}

In the login phase, smart card $S C_{i}$ enables to check the errors immediately with $U_{i}$ 's identity, password, and biometrics, in which flow of login phase is illustrated in the Figure 3 and details are explained as follows.

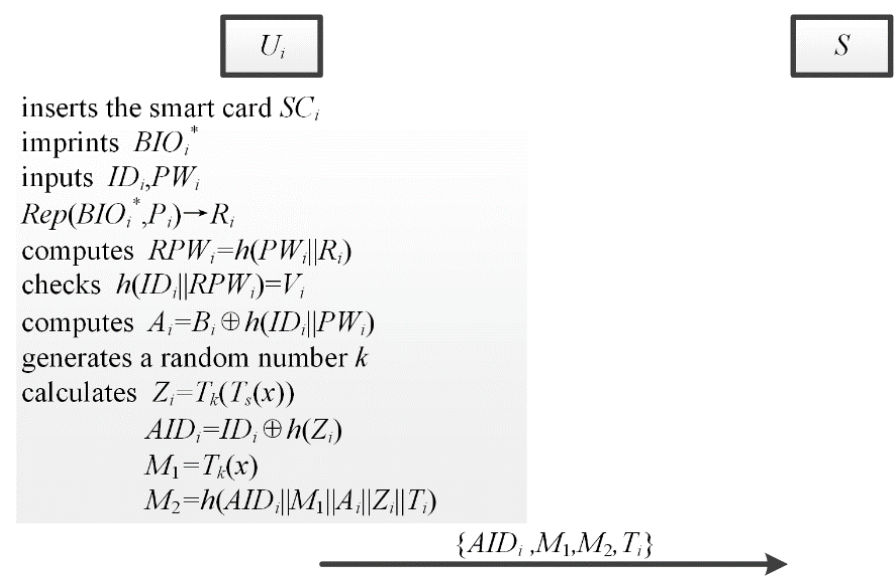

Figure 3. The Login Phase

1. $U_{i}$ first inserts his smart card $S C_{i}$ and imprints his biometrics $B I O_{i}^{*}$, identity $I D_{i}$ and password $P W_{i}$, respectively. Then sensor sketches $U_{i}{ }^{\prime}$ s $B I O_{i}^{*}$ and performs $\operatorname{Rep}\left(B I O_{i}^{*}, P_{i}\right) \rightarrow\left(R_{i},\right)$

2. $S C_{i}$ computes $R P W_{i}=h\left(P W_{i} \| R_{i}\right)$ and needs to check whether $h\left(I D_{i} \| R P W_{i}\right)=V_{i}$ holds. If they matches, $S C_{i}$ further calculates $A_{i}=B_{i} \oplus h\left(I D_{i} \| P W_{i}\right)$. Otherwise, $S C_{i}$ terminates this login.

3. $S C_{i}$ chooses a random number $k$, and calculates $Z_{i}=T_{k}\left(T_{s}(x)\right), A I D_{i}=I D_{i} \oplus h\left(Z_{i}\right)$, $M_{1}=T_{k}(x)$ and $M_{2}=h\left(A I D_{i}\left\|M_{1}\right\| A_{i}\left\|Z_{i}\right\| T_{i}\right)$, in which $T_{i}$ is a timestamp.

4. $S C_{i}$ sends his login request $\left\{A I D_{i}, M_{1}, M_{2}, T_{i}\right\}$ to the server $S$ via a public channel. 


\subsection{Authentication Phase}

During the authentication phase, server $S$ confirms the freshness of login request, in which authentication phase is illustrated in the Figure 4 and details are described as follows.

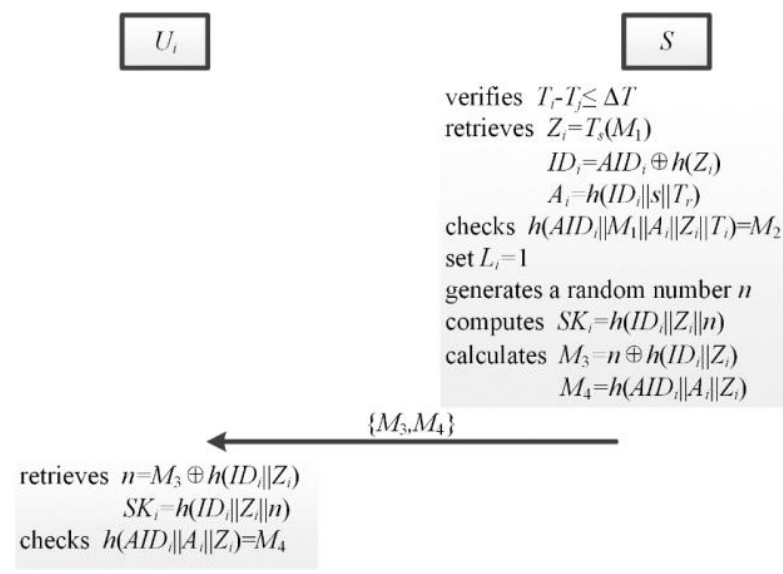

Figure 4. The Authentication Phase

1. Upon receiving $U_{i}$ 's login request, server $S$ needs to verify whether $T_{i}-T_{j} \leq \Delta T$ is valid, in which $\Delta T$ is a time interval and ${ }^{T_{j}}$ is the time when obtaining $U_{i}$ 's login request. If this verification holds, $S$ continues to execute the next step. Otherwise, $U_{i}$ 's login request is rejected by $S$.

2. $S$ retrieves $Z_{i}=T_{s}\left(M_{1}\right), I D_{i}=A I D_{i} \oplus h\left(Z_{i}\right), A_{i}=h\left(I D_{i}\|s\| T_{r}\right)$, respectively, and checks whether $h\left(A I D_{i}\left\|M_{1}\right\| A_{i}\left\|Z_{i}\right\| T_{i}\right)$ is consistent with $M_{2}$.

3. If they matches, $S$ set the login bit ${ }^{L_{i}}$ of status entry to 1 , generates a random number ${ }^{n}$ and computes a session key $S K_{i}=h\left(I D_{i}\left\|Z_{i}\right\| n\right)$. Otherwise, $S$ terminates this authentication.

4. $S$ calculates $M_{3}=n \oplus h\left(I D_{i} \| Z_{i}\right)$ and $M_{4}=h\left(A I D_{i}\left\|A_{i}\right\| Z_{i}\right)$. Then $S$ submits his authentication request $\left\{M_{3}, M_{4}\right\}$ to the user $U_{i}$ via a public channel.

5. After obtaining server $S$ 's authentication request, $S C_{i}$ retrieves $n=M_{3} \oplus h\left(I D_{i} \| Z_{i}\right)$, $S K_{i}=h\left(I D_{i}\left\|Z_{i}\right\| n\right)$ and then verifies whether $h\left(A I D_{i}\left\|A_{i}\right\| Z_{i}\right)=M_{4}$ holds. If they matches, $U_{i}$ adopts this session key ${ }^{S K_{i}}$ to communicate with $S$. Otherwise, authentication is rejected by $U_{i}$.

\subsection{Password Change Phase}

$U_{i}$ is able to update his new password by himself in password change phase, in which details are specified as follows.

1. $U_{i}$ imprints his $B I O_{i}^{*}, I D_{i}$ and $P W_{i}$, respectively. After that, sensor sketches $U_{i}$ 's $B I O_{i}^{*}$ and performs $\operatorname{ReP}\left(B I O_{i}^{*}, P_{i}\right) \rightarrow\left(R_{i},\right)$. 
2. $S C_{i}$ calculates $R P W_{i}=h\left(P W_{i} \| R_{i}\right)$ and then checks whether $h\left(I D_{i} \| R P W_{i}\right)=V_{i}$ holds. If they matches, $U_{i}$ enables to choose a new password. Otherwise, this phase is terminated immediately by $S C_{i}$.

3. $U_{i}$ inputs new password $P W_{i}^{\text {new }}$ and $S C_{i}$ computes $R P W_{i}^{\text {new }}=h\left(P W_{i}^{\text {new }} \| R_{i}\right)$, $B_{i}^{\text {new }}=B_{i} \oplus h\left(I D_{i} \| P W_{i}\right) \oplus h\left(I D_{i} \| P W_{i}^{\text {new }}\right)$ and $V_{i}^{\text {new }}=h\left(I D_{i} \| R P W_{i}^{\text {new }}\right)$, respectively.

4. Finally, $S C_{i}$ replaces $B_{i}$ and $V_{i}$ with $B_{i}^{\text {new }}$ and $V_{i}^{\text {new }}$ in the memory.

\subsection{User Revocation/Re-registration Phase}

When smart card $S C_{i}$ is stolen or lost, user revocation/re-registration enables user $U_{i}$ to revoke his privilege or re-register his account. If $U_{i}$ wants to revoke his privilege, he sends his revocation request to the server $S$ via a secure channel. Next $S$ alters the corresponding array by setting $\left\langle I D_{i}, N_{i}=0, L_{i}\right\rangle$. Similarly, after obtaining $U_{i}$ 's re-registration request, $S$ performs the registration steps which are specified in the registration phase and then replaces $\left\langle I D_{i}, N_{i}=N_{i}+1, L_{i}\right\rangle$ with $\left\langle I D_{i}, N_{i}, L_{i}\right\rangle$ to help $U_{i}$ achieve the re-registration. Thus, user revocation/re-registration makes the presented proposal more robust.

\section{Analysis of our Scheme}

An authentication and key agreement proposal applied for health IoT is expected to perform both security and functionality. In this section, we analysis the performance of our scheme, and compare the proposed scheme with some related proposals.

\subsection{Security Analysis}

We start with the security analysis of the resistance of ours against these common attacks as follows.

6.1.1. Resistance Against Password Guessing Attack: By launching side-channel attacks for instance SPA or DPA, attacker $E$ is able to obtain $B_{i}, P_{i}, V_{i}, x$ and $T_{s}(x)$. However, without information about $B I O_{i}, Z_{i}$ and $s$, this attacker cannot verify $U_{i}$ 's password whether in the on-line or off-line environment. Furthermore, one-way hash function provides a protection for $U_{i}^{\prime}$ 's password, namely, $h\left(P W_{i} \| R_{i}\right)$, where $R_{i}$ has a high entropy. Note that any two users hold different biometric templates due to the uniqueness. Hence, our proposal resists password guessing attack.

6.1.2. Resistance Against Denial-of-Service Attack: DoS attack usually makes server $S$ unavailable, thus server $S$ 's expected capability diminishes dramatically. Server $S$ is able to verify the freshness of $M_{2}=h\left(A I D_{i}\left\|M_{1}\right\| A_{i}\left\|Z_{i}\right\| T_{i}\right)$ by checking the timestamp $T_{i}$ during the authentication phase. If $M_{2}$ is sent by adversary $E$, there is a mismatch due to the wrong timestamp. The presented scheme enables input verification of identity and password, avoiding invalid input or malicious tampering. Moreover, fuzzy extractor is employed in our scheme to satisfy the input requirements of biometric information. In conclusion, our proposal is resilient against DoS attack.

6.1.3. Resistance Against Smart Card Attack: Smart card attacker $E$ attempts to achieve the authentication by applying some information stored in $U_{i}$ 's smart card $S C_{i}$. It 
is unnegligible that $E$ enable to acquire ${ }^{B_{i}}, P_{i}, V_{i}, x$ and $T_{s}(x)$ through SPA or DPA. We present the generation of one session key between user and server as follow.

$$
\begin{gathered}
Z_{i}=T_{s}\left(M_{1}\right), \\
I D_{i}=A I D_{i} \oplus h\left(Z_{i}\right), \\
n=M_{3} \oplus h\left(I D_{i} \| Z_{i}\right), \\
S K_{i}=h\left(I D_{i}\left\|Z_{i}\right\| n\right) .
\end{gathered}
$$

Though acquiring $A I D_{i}, M_{1}$ and $M_{3}$ via a public channel, $E$ is unable to retrieve $Z_{i}, I D_{i}$ and $n$ because of the lack of random numbers $s$ and $k$. As a result, our proposal confines the influence of smart card attack.

6.1.4. Resistance Against User Impersonation Attack: During the user impersonation attack, similar to smart card attack, adversary $E$ impersonates user $U_{i}$ adopting smart card $S C_{i}$ without $U_{i}$ 's password or biometrics. The random numbers $s$ and $k$ are employed to protect $Z_{i}, I D_{i}$ and $n$. Thus, $E$ cannot calculate the session keys even if $E$ obtains ${ }^{B_{i}}, P_{i}$, $V_{i}, x$ and $T_{s}(x)$ by side channel attacks. Consequently, user impersonation attack has no effect on our scheme.

6.1.5. Resistance Against Server Spoofing Attack: After obtaining $U_{i}$ 's login request, adversary $E$ attempts to spoof as server $S$ by replaying old authentication request $\left\{M_{3}^{\text {old }}, M_{4}^{\text {old }}\right\}$, where $M_{3}^{\text {old }}=n^{\text {old }} \oplus h\left(I D_{i} \| Z_{i}^{\text {old }}\right)$ and $M_{4}^{\text {old }}=h\left(A I D_{i}^{\text {old }}\left\|A_{i}\right\| Z_{i}^{\text {old }}\right)$. However, $U_{i}$ applies distinct random numbers for different sessions, namely, $Z_{i}^{\text {old }} \neq Z_{i}^{\text {new }}$. Thus, this attack does not work. Furthermore, due to the inaccessibility of both ${ }^{s}$ and ${ }^{n}, E$ cannot retrieve $Z_{i}$ and $A_{i}$. In conclusion, our proposal prevents server spoofing attack.

6.1.6. Resistance Against Service Misuse Attack: For the service misuse attack, a registered user $E$ intentionally shares some secret parameters to other non-registered users. As a result, each one that knows these parameters, such as identity and password, may login to server $S$ anytime. However, in our improved scheme, $S$ maintains a login bit $L_{i}$ in the status entry for each legal user. Since $L_{i}$ is set to one once a user is logged in, two or more people who know the same registration information cannot to login to $\mathrm{S}$ at the same time. Consequently, the presented scheme performs resistance against service misuse attack.

\subsection{Functionality Analysis}

In previous studies, various functionality requirements for an authentication and key agreement scheme are discussed. We present how our scheme achieves these functionalities during this section.

6.2.1. Anonymity: To perform anonymity, user's real identity cannot be disclosed to another unauthorized party. In our proposal, user $U_{i}$ calculate his dynamic identity ${ }^{A I D_{i}}$ as $A I D_{i}=I D_{i} \oplus h\left(Z_{i}\right)$. Since $Z_{i}$ does not leak out from $U_{i}^{\prime}$ 's request over a public channels, adversary $E$ is unable to compute the user's identity $I D_{i}$. On the other hand, server $S$ can retrieve $Z_{i}=T_{s}\left(M_{1}\right)$, and further calculate $I D_{i}$ from $I D_{i}=A I D_{i} \oplus h\left(Z_{i}\right)$. So the authorized server $S$ is able to confirm $U_{i}$ 's real identity. In conclusion, $E$ keeps unknown to user's real identity, but $U_{i}$ can be authenticated by $S$. 
6.2.2. Session Key Agreement: Through session key agreement, user and server are able to securely establish their session key used for subsequent communication. In our proposal, session key can be calculated by user $U_{i}$ and server $S$ as $S K_{i}=h\left(I D_{i}\left\|Z_{i}\right\| n\right)$, where $Z_{i}$ and ${ }^{n}$ are changeable. Namely, session keys are distinct in different sessions. Hence, previous session keys is difficult to be retrieved by adversary $E$.

6.2.3. Perfect Forward Secrecy: In order to discuss perfect forward secrecy, we give the calculation of a session key as follow.

$$
\begin{gathered}
Z_{i}=T_{s}\left(M_{1}\right), \\
I D_{i}=A I D_{i} \oplus h\left(Z_{i}\right), \\
n=M_{3} \oplus h\left(I D_{i} \| Z_{i}\right), \\
S K_{i}=h\left(I D_{i}\left\|Z_{i}\right\| n\right) .
\end{gathered}
$$

Although user's long-term key $A_{i}$ is compromised, adversary $E$ cannot calculate ${ }^{s}, k$ and $B_{i}$. Thus, this adversary is not able to retrieve $Z_{i}, I D_{i}$ and ${ }^{n}$ to generate a session key between $U_{i}$ and $S$. Consequently, our proposal provides perfect forward secrecy.

6.2.4. Biometric Information Protection: In some previous schemes, $U_{i}$ 's biometrics is directly saved in his smart card $S C_{i}$. In this way, adversary $E$ is able to obtain user's biometric information from smart card through side channel attacks. To avoid this, a high security mechanism is applied in our scheme. We leverages one-way secure hash function to protect a nearly random string $R_{i}$. What is more, this string $R_{i}$ can only be extracted from biometric information ${ }^{B I O_{i}}$ with the help of fuzzy extractor, making it impossible for attacker $E$ to obtain the biometrics. Thus, our scheme achieves biometric information protection.

6.2.5. User Revocation/Re-registration: If user $U_{i}$ wants to revoke his own privilege or re-register, a revocation/re-registration request is required to be sent to server $S$ over a secure channel. Upon receiving this request, $S$ can thereby modify $\left\langle I D_{i}, N_{i}, L_{i}\right\rangle$ in the database to revoke privilege or re-register of user $U_{i}$, which also meets a practical requirement.

6.2.6. Fast Error Detection: Through the fast error detection, smart card $S C_{i}$ is able to verify some discrepancies quickly, for example incorrect passwords, inaccurate identities and false biometrics. During both login and password change phases, $S C_{i}$ can immediately detects the errors without the assistance of server $S$. Therefore, our scheme realizes fast error detection.

\subsection{Comparisons with Related Schemes}

In this section, we concretely compare some properties which includes resistance, functionality and performance between our proposal and other related identity-based authentication schemes, including Lin's scheme [32], Li et al.'s scheme [33] and Wang et al.'s scheme [27].

Table 2 shows the result of resistance comparison among these authenticated key agreement schemes. For simplicity, we define some following notations listed in Table 2, namely, R1: resistance against password guessing attack, R2: resistance against Denialof-Service attack, R3: resistance against smart card attack, R4: resistance against user impersonation attack, R5: resistance against server spoofing attack and R6: resistance 
against service misuse attack. Result indicates that our proposal outperforms other schemes by achieving the requirements of resistance.

Table 2. The Resistance Comparison

\begin{tabular}{|c|c|c|c|c|}
\hline & $\begin{array}{c}\text { Lin's scheme } \\
{[32]}\end{array}$ & $\begin{array}{c}\text { Li et al.'s scheme } \\
{[33]}\end{array}$ & $\begin{array}{c}\text { Wang et al.'s scheme } \\
{[27]}\end{array}$ & $\begin{array}{c}\text { Our } \\
\text { scheme }\end{array}$ \\
\hline R1 & Yes & Yes & Yes & Yes \\
\hline R2 & No & No & No & Yes \\
\hline R3 & Yes & Yes & Yes & Yes \\
\hline R4 & No & No & Yes & Yes \\
\hline R5 & No & Yes & Yes & Yes \\
\hline R6 & Yes & Yes & No & Yes \\
\hline
\end{tabular}

Table 3 specifies the result of functionality comparison among these schemes mentioned above, in which we adopt some following notations, namely, F1: anonymity, F2: session key agreement, F3: perfect forward secrecy, F4: biometric information protection, F5: user revocation/re-registration and F6: fast error detection. It becomes clearly that our proposal provides more functionalities for health IoT, which also makes ours more robust.

Table 3. The Functionality Comparison

\begin{tabular}{|c|c|c|c|c|}
\hline & $\begin{array}{c}\text { Lin's scheme } \\
{[32]}\end{array}$ & $\begin{array}{c}\text { Li et al.'s scheme } \\
{[32]}\end{array}$ & $\begin{array}{c}\text { Wang et al.'s scheme } \\
{[27]}\end{array}$ & $\begin{array}{c}\text { Our } \\
\text { scheme }\end{array}$ \\
\hline F1 & No & Yes & Yes & Yes \\
\hline F2 & No & Yes & No & Yes \\
\hline F3 & No & No & No & Yes \\
\hline F4 & No & No & No & Yes \\
\hline F5 & No & No & No & Yes \\
\hline F6 & No & No & No & Yes \\
\hline
\end{tabular}

Moreover, we compare the presented scheme with above schemes to evaluate the computational costs during login and authentication phases. Note that the computational cost of XOR operation is negligible. Hence, we treat hash function and extended Chebyshev chaotic map as time complexity in the measurement. In Table $4, T_{h}$ denotes the time cost about executing a hash function and $T_{c}$ means the time cost about performing an extended Chebyshev chaotic map. Benefited from Xue et al.'s work [34], processing time of a hash function and an extended Chebyshev chaotic map is $0.2 \mathrm{~ms}$ and $32.2 \mathrm{~ms}$, respectively. As can be seen in Table 4 and Fig. 5, our proposal requires slightly more computation cost than Lin's scheme and Wang et al.'s scheme. But compared to Li et al.'s scheme, our scheme has advantage on computation costs.

Table 4. The Computation Cost Comparison

\begin{tabular}{|c|c|c|c|c|}
\hline & $\begin{array}{c}\text { Lin's scheme } \\
{[32]}\end{array}$ & $\begin{array}{c}\text { Li et al.'s } \\
\text { scheme [33] }\end{array}$ & $\begin{array}{c}\text { Wang et al.'s } \\
\text { scheme [27] }\end{array}$ & $\begin{array}{c}\text { Our } \\
\text { scheme }\end{array}$ \\
\hline $\begin{array}{c}\text { Computational cost } \\
\text { (User) }\end{array}$ & $4 T_{h}+2 T_{c}$ & $8 T_{h}+2 T_{c}$ & $5 T_{h}+2 T_{c}$ & $8 T_{h}+2 T_{c}$ \\
\hline $\begin{array}{c}\text { Execution cost (User) } \\
\begin{array}{c}\text { Computational cost } \\
\text { (Server) }\end{array}\end{array}$ & $65.2 \mathrm{~ms}$ & $66.0 \mathrm{~ms}$ & $65.4 \mathrm{~ms}$ & $66.0 \mathrm{~ms}$ \\
\hline $\begin{array}{c}\text { Execution cost } \\
\text { (Server) }\end{array}$ & $32.8 \mathrm{~ms}$ & $66.2 \mathrm{~ms}$ & $33.0 \mathrm{~ms}$ & $33.4 \mathrm{~ms}$ \\
\hline Total execution cost & $98.0 \mathrm{~ms}$ & $132.2 \mathrm{~ms}$ & $98.4 \mathrm{~ms}$ & $99.4 \mathrm{~ms}$ \\
\hline
\end{tabular}




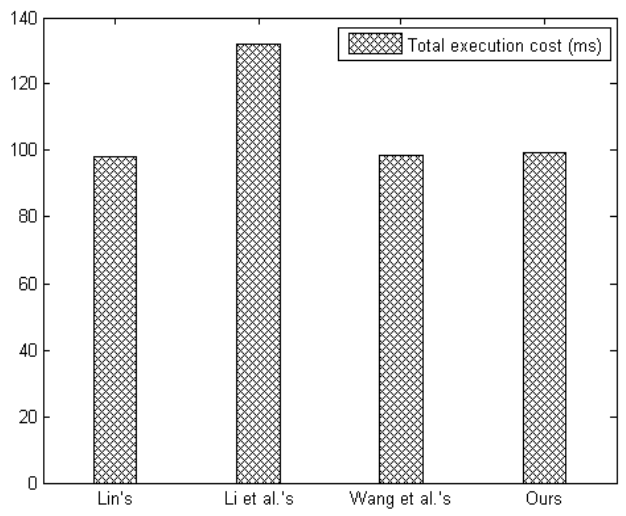

Figure 5. The Computation Cost Comparison

Additionally, we evaluate the performance of our scheme by measuring the communication cost of these methods mentioned above. Without loss of generality, we assume that length of timestamp is 16 bit and the length of some other security parameters is 160 bit, including random number, identity input and hash function output. Hereby, length of login request $\left\{A I D_{i}, M_{1}, M_{2}, T_{i}\right\}$, which user $U_{i}$ submits to server $S$, is $160+160+160+16=496$ bits . And in our authentication phase, communication overhead is $160+160=320$ bits , containing authentication request $\left\{M_{3}, M_{4}\right\}$. As a result, total communication overhead of our proposal is $496+320=816$ bits . We consider the communication overhead of other schemes analogously. As for storage requirement, we estimate the information saved in the smart card $S C_{i}$ and calculate the bit length of stored information as storage overhead. Thus, in our proposal, requirement about stored message $\left\{B_{i}, P_{i}, V_{i}, x, T_{s}(x)\right\}$ contains $160+160+160+160+160=800$ bits . Similarly, we estimate the requirement about storage of other schemes and detailed testing results are presented as below. Table 5 and Fig. 6 show the comparison regarding on both communication cost and storage cost of these schemes. Compared with others, our scheme achieves better efficiency by synthesizing the security, functionality and performance.

Table 5. The Communication and Storage Costs Comparison

\begin{tabular}{|c|c|c|c|c|}
\hline & $\begin{array}{c}\text { Lin's scheme } \\
\text { [32] }\end{array}$ & $\begin{array}{c}\text { Li et al.'s } \\
\text { scheme [33] }\end{array}$ & $\begin{array}{c}\text { Wang et al.'s } \\
\text { scheme [27] }\end{array}$ & $\begin{array}{c}\text { Our } \\
\text { scheme }\end{array}$ \\
\hline $\begin{array}{c}\text { Communication cost } \\
\text { in the login phase }\end{array}$ & $656 \mathrm{bits}$ & 656bits & $496 \mathrm{bits}$ & 496bits \\
\hline $\begin{array}{c}\text { Communication cost } \\
\text { in the authentication phase }\end{array}$ & $176 \mathrm{bits}$ & $832 \mathrm{bits}$ & $176 \mathrm{bits}$ & $320 \mathrm{bits}$ \\
\hline Total communication cost & $832 \mathrm{bit}$ & $1488 \mathrm{bits}$ & $672 \mathrm{bits}$ & $816 \mathrm{bits}$ \\
\hline Storage cost & $480 \mathrm{bits}$ & $960 \mathrm{bits}$ & $480 \mathrm{bits}$ & $800 \mathrm{bits}$ \\
\hline
\end{tabular}




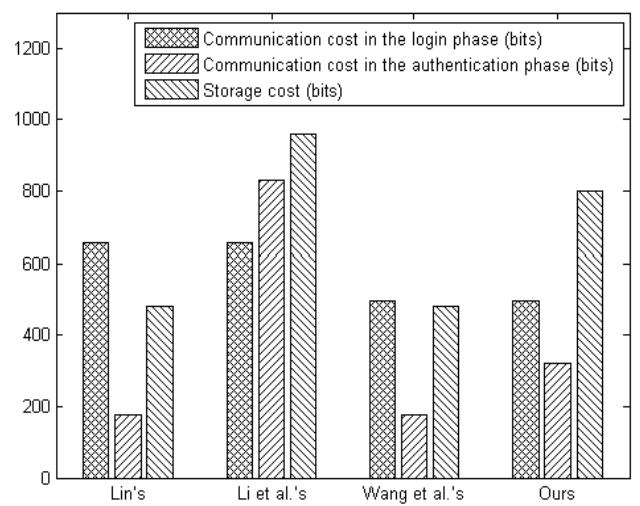

Figure 6. The Communication and Storage Costs Comparison

\section{Conclusion}

This paper has proposed a robust three-factor identity-based authentication and key agreement scheme based on extended chaotic maps for health IoT. According to our comprehensive cryptanalysis, our scheme guarantees effective defense to typical security menaces. Besides, the presented proposal realizes additional significant features than most previous schemes, namely, biometric information protection and user revocation/reregistration. Under the same level of computation cost, communication overhead and storage requirement, our scheme achieves better security with more functionalities. We conclude that the proposed proposal is secure against known attacks, which is also efficient for health IoT.

\section{Acknowledgments}

This research is supported by the Major Program of National Natural Science Foundation of China (No.: 11290141), the National Natural Science Foundation of China (No.: 61402030), and the Fundamental Research of Civil Aircraft (No.: MJ-F-2012-04).

\section{References}

[1] Z. Y. Sun, X. H. Ji and Y. B. Li, "HSKAS: A Novel Hierarchical Shared Key Authentication Scheme in Wireless Sensor Networks", International Journal of Security and Its Applications, vol. 10, no. 4, (2016), pp. 105-116.

[2] J. Srinivas, S. Mukhopadhyay and D. Mishra, "Secure and Efficient User Authentication Scheme for Multi-gateway Wireless Sensor Networks", Ad Hoc Networks, vol. 54, (2017), pp. 147-169.

[3] S. K. H. Islam, M. K. Khan, M. S. Obaidat and F. T. Bin Muhaya, "Provably Secure and Anonymous Password Authentication Protocol for Roaming Service in Global Mobility Networks Using Extended Chaotic Maps", Wireless Personal Communications, vol. 84, no. 3, (2015), pp. 2013-2034.

[4] Y. R. Lu, L. X. Li, H. P. Peng and Y. X. Yang, "An Enhanced Biometric-based Authentication Scheme for Telecare Medicine Information Systems Using Elliptic Curve Cryptosystem", Journal of Medical Systems, vol. 39, no. 3, (2015), pp. 1-8.

[5] X. Li, J. G. Liao, S. Kumari, W. Liang, F. Wu and M. K. Khan, "A New Dynamic ID-based User Authentication Scheme Using Mobile Device: Cryptanalysis, The Principles and Design", Wireless Personal Communications, vol. 85, no. 1, (2015), pp. 263-288.

[6] C. Q. Wang, X. Zhang and Z. M. Zheng, "Cryptanalysis and Improvement of a Biometric-Based MultiServer Authentication and Key Agreement Scheme”, PLoS One, vol. 11, no. 2, (2016), pp. e0149173.

[7] T. Wan, N. Jiang, J. F. Ma and L. Yang, "Cryptanalysis of a Biometric-based Multi-Server Authentication Scheme", International Journal of Security and Its Applications, vol. 10, no. 2, (2016), pp. $163-170$.

[8] A. K. Das and B. Bruhadeshwar, "An Improved and Effective Secure Password-based Authentication and Key Agreement Scheme Using Smart Cards for the Telecare Medicine Information System", Journal of Medical Systems, vol. 37, no. 5, (2013), pp. 1-17. 
[9] Y. P. Lin, K. H. Wang, B. C. Zhang, Y. Z. Liu and X. Li, "An Enhanced Biometric-Based Three Factors User Authentication Scheme for Multi-server Environments", International Journal of Security and Its Applications, vol. 10, no. 1, (2016), pp. 315-328.

[10] B. J. Huang, M. K. Khan, L. B. Wu, F. T. Bin Muhaya and D. B. He, "An Efficient Remote User Authentication with Key Agreement Scheme Using Elliptic Curve Cryptography”, Wireless Personal Communications, vol. 85 , no. 1, (2015), pp. 225-240.

[11] S. K. H. Islam and G. P. Biswas, "A Pairing-free Identity-based Authenticated Group Key Agreement Protocol for Imbalanced Mobile Networks", Annals of Telecommunications, vol. 67, no. 11-12, (2012), pp. 547-558.

[12] C. H. Ye, Z. G. Xiong, Y. M. Ding, X. M. Zhang, G. W. Wang and F. Xu, "Secure Multimedia Content Distribution for M2M Communication", International Journal of Security and Its Applications, vol. 10, no. 4, (2016), pp. 279-288.

[13] D. H. Lee and N. Park, "Security Enhancement Scheme Supporting Range Queries on Encrypted DB for Secure E-Navigation Era", International Journal of Security and Its Applications, vol. 10, no. 2, (2016), pp. 141-150.

[14] B. Ustaoğlu, "Integrating Identity-based and Certificate-based Authenticated Key Exchange Protocols", International Journal of Information Security, vol. 10, no. 4, (2011), pp. 201-212.

[15] S. K. H. Islam and G. P. Biswas, "Provably Secure and Pairing-free Certificateless Digital Signature Scheme Using Elliptic Curve Cryptography”, International Journal of Computer Mathematics, vol. 90, no. 11, (2013), pp. 2244-2258.

[16] H. B. Yang, J. H. Chen and Y. Y. Zhang, "An Improved Two-party Authentication Key Exchange Protocol for Mobile Environment”, Wireless Personal Communications, vol. 85, no. 3, (2015), pp. 13991409.

[17] S. Kumari, M. K. Khan and R. Kumar, "Cryptanalysis and Improvement of 'a Privacy Enhanced Scheme for Telecare Medical Information Systems", Journal of Medical Systems, vol. 37, no. 4, (2013), pp. 1-11.

[18] H. F. Zhu, "Flexible and Password-authenticated Key Agreement Scheme Based on Chaotic Maps for Multiple Servers to Server Architecture", Wireless Personal Communications, vol. 82, no. 3, (2015), pp. 1697-1718.

[19] C. Q. Wang, X. Zhang and Z. M. Zheng, "An Improved Biometrics Based Authentication Scheme Using Extended Chaotic Maps for Multimedia Medicine Information Systems", Multimedia Tools and Applications, (2016), pp. 1-27.

[20] T. S. Messerges, E. Dabbish and R. H. Sloan, "Examining Smart-card Security Under the Threat of Power Analysis Attacks", IEEE Transactions on Computers, vol. 51, no. 5, (2002), pp. 541-552.

[21] S. K. H. Islam, "Provably Secure Dynamic Identity-based Three-factor Password Authentication Scheme Using Extended Chaotic Maps”, Nonlinear Dynamics, vol. 78, no. 3, (2014), pp. 2261-2276.

[22] M. Zhang, J. Zhang and Y. Zhang, "Remote Three-factor Authentication Scheme Based on Fuzzy Extractors", Security and Communication Networks, vol. 8, no. 4, (2015), pp. 682-693.

[23] C. T. Li and M. S. Hwang, "An Efficient Biometrics-based Remote User Authentication Scheme Using Smart Cards", Journal of Network and Computer Applications, vol. 33, no. 1, (2010), pp. 1-5.

[24] X. Li, J. W. Niu, J. Ma, W. D. Wang and C. L. Liu, "Cryptanalysis and Improvement of a BiometricsBased Remote User Authentication Scheme Using Smart Cards", Journal of Network and Computer Applications, vol. 34, no. 1, (2011), pp. 73-79.

[25] Y. Dodis, R. Ostrovsky, L. Reyzin and A. Smith, "Fuzzy Extractors: How to Generate Strong Keys from Biometrics and Other Noisy Data", SIAM Journal on Computing, vol. 38, no. 1, (2008), pp. 75-80.

[26] Y. Dodis, B. Kanukurthi, J. Katz, L. Reyzin and A. Smith, "Robust Fuzzy Extractors and Authenticated Key Agreement from Close Secrets", IEEE Transactions on Information Theory, vol. 58, no. 9, (2012), pp. 6207-6222.

[27] Z. H. Wang, Z. Q. Huo and W. B. Shi, "A Dynamic Identity Based Authentication Scheme Using Chaotic Maps for Telecare Medicine Information Systems”, Journal of Medical Systems, vol. 39, no. 1, (2015), pp. 1-8.

[28] P. Bergamo, P. D'Arco, A. De Santis and L. Kocarev, "Security of Public-key Cryptosystems Based on Chebyshev Polynomials", IEEE Transactions on Circuits and Systems-I: Regular Papers, vol. 52, no. 7, (2005), pp. 1382-1393.

[29] L. Zhang, "Cryptanalysis of the Public Key Encryption Based on Multiple Chaotic Systems", Chaos, Solitons \& Fractals, vol. 37, no. 3, (2008), pp. 669-674.

[30] T. F. Lee, "Verifier-based Three-party Authentication Schemes Using Extended Chaotic Maps for Data Exchange in Telecare Medicine Information Systems", Computer Methods and Programs in Biomedicine, vol. 117, no. 3, (2014), pp. 464-472.

[31] D. Dolev and A. Yao, "On the Security of Public Key Protocols", IEEE Transactions on Information Theory, vol. 29, no. 2, (1983), pp. 198-208.

[32] H. Lin, "Chaotic Map Based Mobile Dynamic ID Authenticated Key Agreement Scheme", Wireless Personal Communications, vol. 78, no. 2, (2014), pp. 1487-1494. 
[33] C. T. Li, C. L. Cheng and Y. W. Chi, "A Secure Chaotic Maps and Smart Cards Based Password Authentication and Key Agreement Scheme with User Anonymity for Telecare Medicine Information Systems", Journal of Medical Systems, vol. 38, no. 9, (2014), pp. 1-11.

[34] K. P. Xue and P. L. Hong, "Security Improvement on an Anonymous Key Agreement Protocol Based on Chaotic Maps", Communications in Nonlinear Science and Numerical Simulation, vol. 17, no. 7, (2012), pp. 2969-2977.

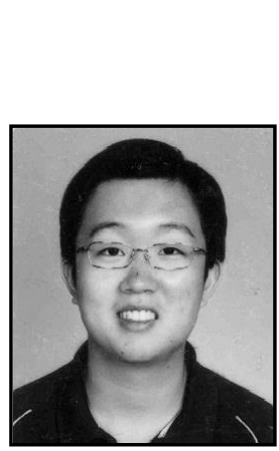

\begin{abstract}
Authors
Chengqi Wang, received the B.S. degree with distinction from Beihang University, Beijing, China, in 2012. He was a Ph.D. candidate at Key Laboratory of Mathematics, Informatics and Behavioral Semantics and School of Mathematics and Systems Science, Beihang University. He received the Ph.D degree from Beihang University, Beijing, China, in 2017. His research interests include network security and applied cryptography.
\end{abstract}

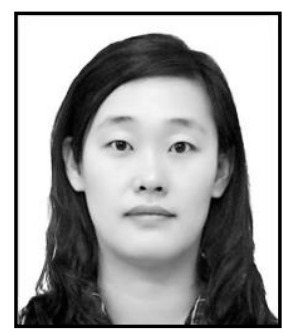

Xiao Zhang, received the Ph.D degree from Beihang University, Beijing, China, in 2013. She is currently the associate professor of Mathematics at Beihang University and the member of Key Laboratory of Mathematics, Informatics and Behavioral Semantics, Ministry of Education. Her research interests include cryptography, information security and complex information system.

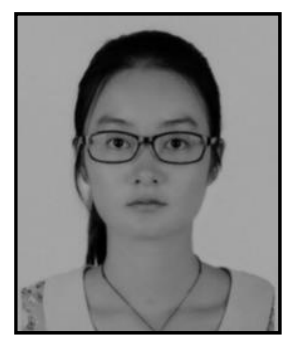

Lijia Xie, received the B.S. degree with distinction from Beihang University, Beijing, China, in 2016. She is currently a Master student at Key Laboratory of Mathematics, Informatics and Behavioral Semantics and School of Mathematics and Systems Science, Beihang University. Her research interests include network security and applied cryptography.

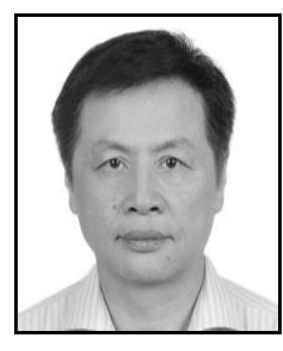

Zhiming Zheng, received the Ph.D. degree from Peking University, Beijing, China, in 1987. He is currently the Professor of Mathematics at Beihang University and the Director of Key Laboratory of Mathematics, Informatics and Behavioral Semantics, Ministry of Education. His research interests include information security, complex information system, and dynamic system. He is the Editor in Chief of the journal Mathematical Biosciences and Engineering published by SPRINGER, and the journal Mathematics in Computer Science published by BIRKHAUSER. 
International Journal of Security and Its Applications

Vol. 11, No. 9 (2017) 